\title{
Novos registros de Lutzomyia longipalpis (Lutz \& Neiva, 1912) (Diptera: Psychodidae) na regiáo Centro-Leste do estado de Sáo Paulo, Brasil
}

\author{
New records of Lutzomyia longipalpis (Lutz \& Neiva, 1912) (Diptera: Psychodidae) \\ in the Central East Region of São Paulo State, Brazil \\ André Antonio Cutolo ${ }^{1 *}$; Denis Adriano Camargo ${ }^{2}$; Claudio José Von Zuben ${ }^{3}$
}

${ }^{1}$ Departamento de Parasitologia, Instituto de Biologia, Universidade Estadual de Campinas - UNICAMP

${ }^{2}$ Curso de Ciências Biológicas, Centro Universitário Hermínio Ometto - UNIARARAS

${ }^{3}$ Departamento de Zoologia, Instituto de Biociências, Universidade Estadual Paulista - UNESP

Recebido em 7 de Novembro de 2008

Aceito em 11 de Dezembro de 2008

\section{Resumo}

A incidência das leishmanioses tegumentar (LTA) e visceral (LVA) americanas, especialmente essa última, em hospedeiros caninos e humanos, encontra-se em expansão no Estado de São Paulo. Na vigilância epidemiológica dessas endemias, torna-se fundamental o conhecimento da distribuição e ecologia das diferentes espécies de flebotomíneos. Assim, a divulgação de novos registros de seus vetores é fundamental para apontar novas áreas de risco para a transmissão dessas doenças. Neste estudo, realizaram-se capturas de flebotomíneos em ambiente de mata, em diferentes localidades dos municípios de Ipeúna, Itirapina e Analândia, entre agosto e setembro de 2007. Foram capturados 248 flebotomíneos de nove espécies diferentes, em Ipeúna, seis e sete espécimes de duas espécies distintas coletados respectivamente em Itirapina e Analândia. A espécie mais abundante em Ipeúna foi Pintomyia pessoai (37,5\%), seguida de P. fischeri (33,06\%) e Migonemyia migonei (16,53\%). Essas três espécies são consideradas importantes vetores de LTA no território paulista. O registro de Lutzomyia longipalpis pela primeira vez em Ipeúna e Analândia e a confirmaçáo de sua presença em Itirapina indicam risco de essabelecimento da LVA na área e a necessidade de mais estudos locais sobre sua ecologia, sobretudo em relação à ocupação de ambientes antrópicos.

Palavras-chave: vetores de leishmaniose, ecologia de vetores, epidemiologia, transmissão, flebotomíneo.

\begin{abstract}
Cutaneous (LTA) and Visceral (LVA) American Leishmaniasis incidences are increasing in human and canine hosts, especially LVA, which is expanding its range through São Paulo State. Distribution and ecology knowledge of different sand fly species is essential for leishmaniasis epidemiology vigilance. The communication of new findings of its vectors is mandatory for risk determination for transmission of these illnesses. On this study sand flies were trapped in bushed areas, in different localities at rural areas of Ipeúna, Itirapina and Analândia counties, between August and September 2007. A total of 248 specimens of nine different species were sampled in Ipeúna, six and seven specimens of two different species were trapped in Itirapina and Analândia, respectively. The most abundant species in Ipeúna was Pintomyia pessoai (37.5\%), followed by Pintomyia fischeri (33.06\%) and Migonemyia migonei (16.53\%), all three species are considered vectors of LTA in São Paulo State. Lutzomyia longipalpis was found in Ipeúna and Analândia for the first time and its presence confirmed in Itirapina, indicating a risk of LVA essablishment in the area and the need for further local studies on its ecology, especially regarding its occupation of the anthropic environment.
\end{abstract}

Keywords: leishmaniasis vector, epidemiology, transmission, zoonosis, sand flies.

A Leishmaniose Visceral Americana - LVA é uma zoonose em franca expansão no estado de São Paulo. De 1999 até dezembro de 2008, foram identificados pela Secretaria Estadual de Saúde, através do Centro de Vigilância Epidemiológica, casos

\footnotetext{
*Autor para correspondência: André Antonio Cutolo

Departamento de Parasitologia, Instituto de Biologia, Universidade Estadual

de Campinas - UNICAMP, Cidade Universitária Zeferino Vaz, s/n,

CEP 13083-970, Campinas - SP, Brasil; e-mail: cutoloandre@yahoo.com
}

humanos de Leishmaniose Visceral Americana em 54 municípios, prevalecendo os do oeste paulista, num total de $1.378 \mathrm{ca}-$ sos, sendo 122 fatais, apresentando letalidade de 8,85\% (CVE, 2008). Nessa região, 267.383 cáes foram avaliados por sorologia de 1999 até 2004, sendo 18.513 (6,92\%) considerados positivos para a infecção (CVE, 2007). Das 645 cidades do Estado de São Paulo, 63 (5,8\%) registraram casos de LVA humana e/ou 
canina até fevereiro de 2008 (GRUPO DE ESTUDOS DE LEISHMANIOSES - CCD, 2008).

Lutzomyia longipalpis (Lutz \& Neiva, 1912) é considerado o principal vetor da LVA no Brasil (LAINSON; RANGEL, 2003), e o conhecimento da sua distribuição no território paulista é fundamental para o controle dessa enfermidade. Inquéritos entomológicos no estado de Sáo Paulo mostraram a presença dessa espécie vetora no ambiente intradomiciliar no município de Araçatuba (COSTA et al., 1997). Posteriormente, foram identificados 361 municípios $(55,9 \%$ do total de municípios do estado) que se apresentavam receptivos para a doença, com presença de L. longipalpis (GRUPO DE ESTUDOS DE LEISHMANIOSES - CCD, 2008).

No presente estudo, as atividades de levantamento de flebotomíneos foram realizadas nos municípios de Ipeúna, Itirapina e Analândia (Figura 1). Em Ipeúna e Itirapina, os pontos de captura situavam-se na Cuesta Basáltica da Serra de Itaqueri e, em Analândia, em morros testemunhos também associados às Cuestas Basálticas.

Nas Cuestas Basálticas, o relevo é escarpado, predominando declividades altas (maiores que 30\%) e amplitudes maiores que $100 \mathrm{~m}$ (PONÇANO et al., 1981). Todas as coletas foram realizadas em área de mata, próximas a afloramentos rochosos areníti- co-basálticos. Os locais avaliados também estão associados a paisagens e elementos de marcante beleza natural, como cavernas, morros e escarpas, com relevante interesse turístico (Figura 2).

Para a captura dos insetos, armadilhas automáticas luminosas do tipo CDC foram posicionadas a uma distância de 50 a $80 \mathrm{~cm}$ do solo e funcionaram por um período ininterrupto das 18 às 8 horas da manhã do dia seguinte. Em 2007, foram realizadas duas coletas em Ipeúna, utilizando-se duas armadilhas, uma na mata defronte à abertura da Caverna do Fazendão e outra em paredão de arenito, próximo à mesma caverna (22 29' $24,5^{\circ}$ ' S e $47^{\circ} 38^{\prime} 42,7^{\prime \prime}$ O e $529 \mathrm{~m}$ de altitude), nos dias 12 de agosto e 2 de setembro; duas coletas em Itirapina utilizando-se uma armadilha na mata defronte à abertura da Caverna Boca do Sapo (22 $25^{\prime} 04,7^{\prime \prime} \mathrm{S}$ e $47^{\circ} 47^{\prime} 32,8^{\prime \prime} \mathrm{O}$ e $808 \mathrm{~m}$ de altitude), nos dias 19 de agosto e 23 de setembro, e uma coleta em Analândia utilizando-se três armadilhas, sendo uma colocada na mata próxima

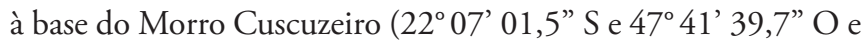
$869 \mathrm{~m}$ de altitude); e as outras duas, colocadas respectivamente na base ( $22^{\circ} 06^{\prime} 38,7^{\prime \prime} \mathrm{S}$ e $47^{\circ} 41^{\prime} 22,6^{\prime \prime} \mathrm{O}$ e $916 \mathrm{~m}$ de altitude) e no topo (22 $06^{\prime} 39,1^{\prime \prime} \mathrm{S}$ e $47^{\circ} 41^{\prime} 24^{\prime \prime} \mathrm{O}$ e $935 \mathrm{~m}$ de altitude) do Morro do Camelo, em meio à mata, no dia 7 de setembro.

As coordenadas dos locais de captura foram obtidas por meio de aparelho GPS Garmin Etrex ${ }^{\oplus}$ (Global Positioning System) e,

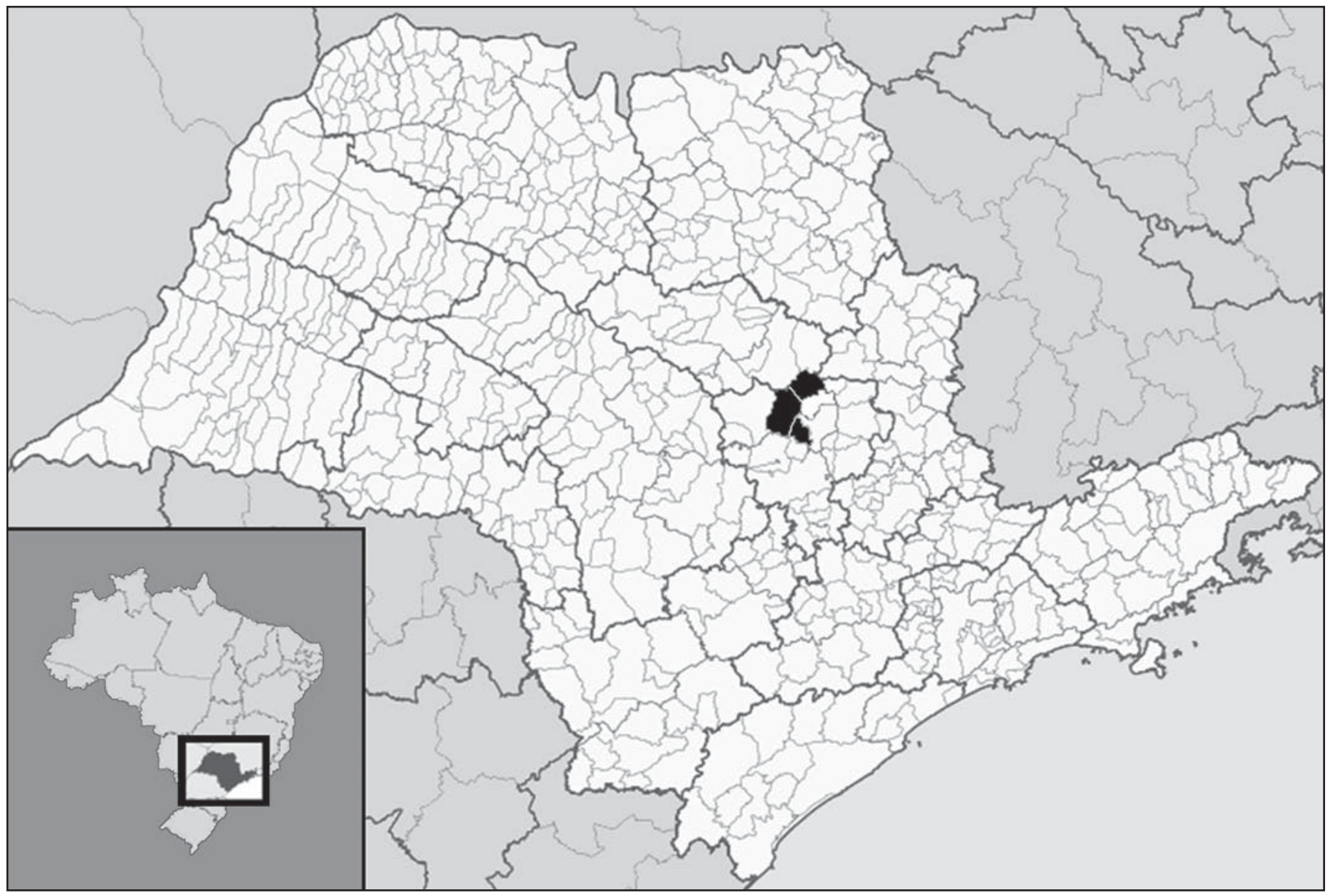

Figura 1. Localização dos municípios de Analândia, Itirapina e Ipeúna em negro, na região Centro-Leste do estado de São Paulo, de cima para baixo respectivamente no mapa. 
Tabela 1. Flebotomíneos capturados em armadilhas luminosas do tipo CDC em área de mata defronte à entrada da Caverna do Fazendão e Paredão de Arenito, município de Ipeúna, Estado de São Paulo, Brasil, por espécie, sexo e local da armadilha, nos meses de Agosto e Setembro de 2007.

\begin{tabular}{|c|c|c|c|c|c|c|c|c|}
\hline \multirow{2}{*}{ Espécie } & \multicolumn{2}{|c|}{ Mata defronte caverna } & \multicolumn{2}{|c|}{ Paredáo de arenito } & \multicolumn{2}{|c|}{ Sub-total } & \multicolumn{2}{|c|}{ Total } \\
\hline & $\mathbf{F}$ & $\mathbf{M}$ & $\mathbf{F}$ & $\mathbf{M}$ & $\mathbf{F}$ & $\mathbf{M}$ & $\mathbf{F}+\mathbf{M}$ & $\%$ \\
\hline Pintomyia pessoai & 68 & 24 & 0 & 1 & 68 & 25 & 93 & 37,50 \\
\hline Pintomyia fischeri & 24 & 56 & 2 & 0 & 26 & 56 & 82 & 33,06 \\
\hline Migonemyia migonei & 37 & 4 & 0 & 0 & 37 & 4 & 41 & 16,53 \\
\hline Expapillata firmatoi & 8 & 0 & 1 & 0 & 16 & 2 & 18 & 7,26 \\
\hline Brumptomyia nitzulescui & 1 & 4 & 0 & 0 & 1 & 4 & 5 & 2,02 \\
\hline Psathyromyia shannoni & 3 & 0 & 0 & 0 & 3 & 0 & 3 & 1,21 \\
\hline Lutzomyia longipalpis & 2 & 0 & 1 & 0 & 3 & 0 & 3 & 1,21 \\
\hline Nyssomyia whitmani & 2 & 0 & 0 & 0 & 2 & 0 & 2 & 0,81 \\
\hline Pintomyia monticola & 1 & 0 & 0 & 0 & 1 & 0 & 1 & 0,40 \\
\hline Total & 146 & 88 & 4 & 1 & 157 & 91 & 248 & 100 \\
\hline$\%$ & 58,87 & 35,48 & 1,61 & 0,40 & 63,31 & 36,69 & 100 & \\
\hline
\end{tabular}

F: fêmeas; M: machos; F + M: soma de fêmeas e machos.

posteriormente, plotadas em mapas topográficos da região. Neste estudo, totalizaram-se cinco noites de coleta, resultando em 126 horas de captura. A identificação dos insetos foi feita segundo Galati (2003).

Nove espécies diferentes foram capturadas, considerandose os três municípios. As nove estiveram presentes em Ipeúna, com dominância de Pintomyia pessoai que se mostrou a espécie mais abundante com 93 espécimes (37,5\%), seguida por P. fischeri, com 82 espécimes (33,06\%), por Migonemyia migonei com 41 espécimes $(16,53 \%)$ e por Expapillata firmatoi, com 18 (7,26\%) indivíduos capturados. As outras cinco espécies capturadas corresponderam a aproximadamente $6 \%$ do total e incluem importantes espécies vetoras, como Nyssomyia whitmani e Lutzomyia longipalpis, conforme apresentado na Tabela 1.

Em Itirapina e Analândia, embora a quantidade de insetos capturados tenha sido marcadamente menor, quando comparada às capturas de Ipeúna, L. longipalpis mostrou-se a espécie dominante, conforme apresentado nas Tabelas 2 e 3.

Expapillata firmatoi é uma espécie antropofílica e já foi assinalada picando o homem durante o dia (GOMES, 1994). Pintomyia pessoai, P. fischeri, M. migonei, $N$. whitmani são consideradas importantes vetores de LTA, no estado de São Paulo, (GOMES, 1994; CAMARGO-NEVES et al., 2002); e L. longipalpis a principal transmissora de LVA no Brasil (LAINSON; RANGEL, 2003; MINISTÉRIO DA SAÚDE, 2003). A presença de L. longipalpis já havia sido constatada no mesmo local de captura, em Itirapina (CUTOLO et al., 2008), e se confirma com o presente achado de indivíduos machos dessa espécie. A espécie foi encontrada pela primeira vez nos municípios de Ipeúna e Analândia, o que os classifica, segundo o Manual de Controle da Leishmaniose Visceral Americana (MINISTÉRIO DA SAÚDE, 2003), como receptivos para essa grave endemia.

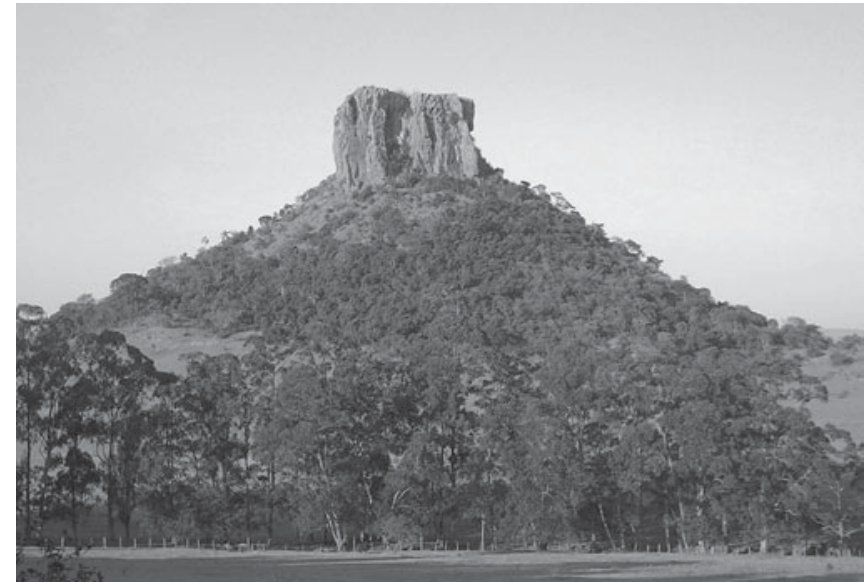

Figura 2. Relevo escarpado com afloramentos rochosos areníticobasálticos associados à mata nativa, habitat de Lutzomyia longipalpis. Morro do Cuscuzeiro, município de Analândia, estado de São Paulo.

Tabela 2. Flebotomíneos capturados em armadilhas luminosas do tipo CDC em área de mata defronte à entrada da Caverna Boca do Sapo, município de Itirapina, estado de São Paulo, Brasil, por espécie e sexo, no mês de Setembro de 2007.

\begin{tabular}{lcccccc}
\hline \multirow{2}{*}{ Espécie } & \multicolumn{3}{c}{ Mata defronte caverna } & & \multicolumn{2}{c}{ Total } \\
\cline { 2 - 3 } \cline { 6 - 7 } & $\mathbf{F}$ & $\mathbf{M}$ & & $\mathbf{F}+\mathbf{M}$ & $\%$ \\
\hline Lutzomyia longipalpis & 1 & 4 & & 5 & 83,33 \\
Pintomyia monticola & 1 & 0 & & 1 & 16,67 \\
Total & 2 & 4 & & 6 & 100 \\
$\%$ & 33,33 & 66,67 & & 100 & \\
\hline
\end{tabular}

F: fêmeas; M: machos; F + M: soma de fêmeas e machos. 
Tabela 3. Flebotomíneos capturados em armadilhas luminosas do tipo CDC em áreas de mata nos Morros Cuscuzeiro e do Camelo, município de Analândia, estado de São Paulo, Brasil, por espécie, sexo e local da armadilha, no mês de Setembro de 2007.

\begin{tabular}{|c|c|c|c|c|c|c|c|c|}
\hline \multirow{2}{*}{ Espécie } & \multicolumn{2}{|c|}{ Morro Cuscuzeiro } & \multicolumn{2}{|c|}{ Morro do Camelo } & \multicolumn{2}{|c|}{ Sub-total } & \multicolumn{2}{|c|}{ Total } \\
\hline & $\mathbf{F}$ & M & $\mathbf{F}$ & $\mathbf{M}$ & $\mathbf{F}$ & M & $F+M$ & $\%$ \\
\hline Lutzomyia longipalpis & 3 & 1 & 2 & 0 & 5 & 1 & 6 & 85,72 \\
\hline Migonemyia migonei & 0 & 0 & 1 & 0 & 1 & 0 & 1 & 14,28 \\
\hline Total & 3 & 1 & 3 & 0 & 6 & 1 & 7 & 100 \\
\hline$\%$ & 42,86 & 14,28 & 42,87 & 0 & 85,71 & 14,28 & 100 & \\
\hline
\end{tabular}

F: fêmea; M: macho; F + M: soma de fêmeas e machos.

A dominância de $P$. pessoai em Ipeúna deve-se provavelmente à preservação da mata no local de captura. Tal fato é reforçado pela total ausência de Nyssomyia neivai, considerado o principal vetor de LTA no território paulista e associado a áreas antropizadas com vegetação alterada (CAMARGO-NEVES et al., 2002). P. fischeri, N. whitmani e $M$. migonei estáo presentes tanto em áreas de matas preservadas como de vegetação alterada (GOMES, 1994), e, em especial essas duas últimas, poderiam assim carrear Leishmania spp. de um ciclo enzoótico para o peridomiciliar quando infectadas.

As espécies encontradas no município de Ipeúna ( $P$. pessoai, P. fischeri, $M$. migonei e $N$. whitmani) e Analândia ( $M$. migonei) indicam estes ambientes como importantes locais de risco para a transmissão de LTA.

O encontro de $L$. longipalpis em área de mata nos municípios de Ipeúna, Analândia e a confirmação de sua presença em Itirapina, associada às Cuestas Basálticas com afloramentos rochosos arenítico-basálticos, mostram um habitat silvestre da espécie na região. A importância turística dos locais avaliados reforça a necessidade de se avaliar a existência de infecção natural de flebotomíneos por Leishmania spp., identificando-se assim o risco de aquisição de leishmanioses ao se visitar tais áreas.

Face à expansão da LVA no Estado, faz-se necessária e urgente uma avaliação da possibilidade de L. longipalpis dispersarse para ambientes antrópicos, em locais onde a forma de relevo da região estudada no presente trabalho está presente, pois as Cuestas Basálticas cortam transversalmente o centro do Estado de São Paulo, indo do Rio Grande até o Rio Paranapanema, sendo umas das mais marcantes feiçóes do relevo paulista, caracterizadas pela presença de relevo escarpado nos limites com a Depressão Periférica (PONÇANO et al., 1981).

\section{Referências}

CAMARGO-NEVES, V. L. F; GOMES, A. C.; ANTUNES, J. L. F. Correlação da presença de espécies de flebotomíneos (Diptera: Psychodidae) com registros de casos de leishmaniose tegumentar americana no Estado de São Paulo, Brasil. Revista da Sociedade Brasileira de Medicina Tropical, v. 35, n. 4, p. 299-306, 2002.
CENTRO DE VIGILÂNCIA EPIDEMIOLÓGICA "PROF. ALEXANDRE VRANJAC” - CVE. Divisão de Zoonoses. Secretaria de Estado da Saúde. Disponível em: <http://www.cve.saude.sp.gov.br>. Acesso em: 25 de Dezembro de 2008.

COSTA, A. I. P.; CASANOVA, C.; RODAS, L. A. C.; GALATI, E. A. B. Atualização da distribuição geográfica e primeiro encontro de Lutzomyia longipalpis em área urbana no Estado de São Paulo, Brasil. Revista de Saúde Pública, v. 31, n. 6, p. 632-633, 1997.

CUTOLO, A. A.; CAMARGO, D. A.; CUTOLO, A. A.; VON ZUBEN, C. J.; GALATI, E. A. B. Lutzomyia longipalpis (Diptera, Psychodidae) em Cuessa Basáltica na bacia hidrográfica do Rio Corumbataí, Região Centro-leste do Estado de São Paulo. Revista Brasileira de Epidemiologia, v. 11, n. 2, p. 336-339, 2008.

Divisão de Zoonoses. Secretaria de Estado da Saúde. Disponível em: <http://www.cve.saude.sp.gov.br>. Acesso em: 15 de Novembro de 2007.

GALATI, E. A. B. Morfologia, terminologia de adultos e identificação dos táxons da América. In: RANGEL, E. F.; LAINSON, R. (Eds.). Flebotomíneos do Brasil. Rio de Janeiro: Fiocruz, 2003. p. 53-175.

GOMES, A. C. Sand fly vectorial ecology in the State of São Paulo. Memórias do Instituto Oswaldo Cruz, v. 89, n. 3, p. 457-60, 1994.

GRUPO DE ESTUDOS EM LEISHMANIOSES, CCD. Atualização da classificação epidemiológica dos municípios para a Leishmaniose Visceral Americana. Boletim Epidemiológico Paulista, v. 5, n. 50, p. 18-25, 2008.

LAINSON, R; RANGEL, E. Lutzomyia longipalpis e a eco-epidemiologia da leishmaniose visceral americana (LVA) no Brasil. In: RANGEL, E. F; LAINSON, R. (Eds.). Flebotomíneos do Brasil. Rio de Janeiro: Fiocruz, 2003. p. 311-336.

MINISTÉRIO DA SAÚDE. Secretaria de Vigilância em Saúde. Departamento de Vigilância Epidemiológica. Manual de Vigilância e Controle da Leishmaniose Visceral. Brasília, 2003. 120 p.

PONÇANO, W. L.; CARNEIRO, C. D. R.; BISTRICHI, C. A.; ALMEIDA, F. F. M.; PRANDINI, F. L. Mapa Geomorfológico do Estado de Sáo Paulo. Vol. 1. São Paulo: Divisão de Minas e Geologia Aplicada, Instituto de Pesquisas Tecnológicas do Estado de São Paulo, 1981.37 p. 